\title{
Left atrial hypertension and the risk of early incident heart failure after atrial fibrillation ablation
}

\author{
Jasen Gilge ${ }^{1}$, Asim Ahmed ${ }^{2}$, Brad Clark ${ }^{2}$, Alexander Slaten ${ }^{1}$, Rahul Devathu ${ }^{1}$, Jeff Olson ${ }^{3}$, \\ Benzy Padanilam ${ }^{2}$, Girish Nair ${ }^{4}$, Sandeep Joshi ${ }^{1}$, Ashwin Ravichandran ${ }^{4}$, and Parin Patel ${ }^{1}$ \\ ${ }^{1}$ Ascension St Vincent Hospital Indianapolis \\ ${ }^{2}$ St. Vincent Health \\ ${ }^{3}$ St Vincent \\ ${ }^{4}$ St. Vincent Medical Group
}

September 21, 2020

\begin{abstract}
Introduction: Elevated left atrial pressure (LAP) during catheter ablation of atrial fibrillation (AF) is associated with an increased risk of AF recurrence, but it is unknown if this correlates with heart failure (HF). The objective of the study was to determine if elevated LAP after AF ablation correlates with HF events. Methods: Prospective, single center, cohort study measuring LAP and RAP during AF ablation in 100 patients. The primary endpoint was clinical HF within 30 days of ablation. The secondary outcome was AF-free HF. Results: 100 patients (63\% male, mean age 64.5 ) were enrolled and $20 \%$ had clinical HF within 30 days. Bivariate correlates included mitral valve (MV) disease, persistent AF, class III antiarrhythmics, LAP, and recurrent AF. Multivariate analysis revealed class III antiarrhythmics were protective (OR 0.24 [0.1-0.5], p=0.04), while MV disease (OR 8.7 [3.3-23], $\mathrm{p}=0.03$ ) and loop diuretics (OR 4.8 [2.6-9.1], $\mathrm{p}=0.01$ ) were hazardous. AF-free HF occurred in 9\% of patients and correlated with higher LAP and RAP, and CKD. Conclusion: Patients with HF after AF ablation had higher LAP. MV disease, diuretic use, and class III antiarrhythmics also correlated to HF. These present opportunities to target future interventions to reduce a common complication of AF ablation.
\end{abstract}

Left atrial hypertension and the risk of early incident heart failure after atrial fibrillation ablation

Jasen L Gilge MD ${ }^{\mathrm{a}}$; Asim Ahmed $\mathrm{DO}^{\mathrm{a}}$; Brad A Clark DO ${ }^{\mathrm{a}}$; Alexander Slaten DO ${ }^{\mathrm{a}}$; Rahul Devathu; Jeff A Olson DO ${ }^{\mathrm{a}}$; Benzy J Padanilam MD ${ }^{\mathrm{a}}$; Girish V Nair MD ${ }^{\text {a }}$; Sandeep A Joshi MD ${ }^{\mathrm{a}}$; Ashwin K Ravichandran $\mathrm{MD}^{\mathrm{a}}$; Parin J Patel MD

\section{Corresponding Author:}

Jasen Gilge, M.D.

8333 Naab Road, \#400

Indianapolis, IN 46260

Phone: 317.338.6666, FAX: 317.338.6457

e-mail: jasen.gilge@ascension.org

\section{All Authors are from the}

St Vincent Medical Group, 8333 Naab Road, \#400,Indianapolis, IN 46260. Department of Internal Medicine, Division of Cardiology 
Conflict of interest : Parin Patel serves on an advisory panel for Medtronic, Inc. Ashwin Ravichandran reports speaking honoraria and travel support from Abbott and Medtronic. The remaining authors have no conflicts to disclose.

Financial disclosure : None

\section{ABSTRACT}

Introduction: Elevated left atrial pressure (LAP) during catheter ablation of atrial fibrillation (AF) is associated with an increased risk of AF recurrence, but it is unknown if this correlates with heart failure (HF). The objective of the study was to determine if elevated LAP after AF ablation correlates with HF events.

Methods: Prospective, single center, cohort study measuring LAP and RAP during AF ablation in 100 patients. The primary endpoint was clinical HF within 30 days of ablation. The secondary outcome was AF-free HF.

Results: 100 patients (63\% male, mean age 64.5) were enrolled and $20 \%$ had clinical HF within 30 days. Bivariate correlates included mitral valve (MV) disease, persistent AF, class III antiarrhythmics, LAP, and recurrent AF. Multivariate analysis revealed class III antiarrhythmics were protective (OR 0.24 [0.1-0.5], $\mathrm{p}=0.04$ ), while MV disease (OR 8.7 [3.3 - 23], $\mathrm{p}=0.03$ ) and loop diuretics (OR 4.8 [2.6-9.1], $\mathrm{p}=0.01$ ) were hazardous. AF-free HF occurred in $9 \%$ of patients and correlated with higher LAP and RAP, and CKD.

Conclusion: Patients with HF after AF ablation had higher LAP. MV disease, diuretic use, and class III antiarrhythmics also correlated to HF. These present opportunities to target future interventions to reduce a common complication of AF ablation.

Key Words : Atrial fibrillation, ablation, congestive heart failure

Word Count : 4130

\section{Abbreviations}

Atrial fibrillation (AF)

Heart Failure (HF)

Right Atrial Pressure (RAP)

Left Atrial Pressure (LAP)

Chronic Kidney Disease (CKD)

Catheter Ablation (CA)

INTRODUCTION

Atrial fibrillation is the commonest arrhythmia worldwide, and is projected to increase in frequency as the population ages. (1,2) The cornerstone of AF management is symptom control, and multiple studies suggest catheter ablation is a safe and effective procedure to reduce symptoms from AF. (3,4) Procedure efficacy has remained the same, but newer techniques and technologies are making for a safer procedure. Nevertheless, one major complication is incident HF requiring office visits, medicine titration, emergency room visits, or hospitalization, which can be as high as $20 \%$. (5-10)

Atrial fibrillation and HF frequently coexist in this population, with AF prevalence ranging from $5 \%$ in patients with mild HF up to $50 \%$ in patients with severe HF. (11) These disease processes share numerous risk factors such as obesity, hypertension and diabetes, resulting in adverse cellular remodeling, neurohormonal changes and increased LAP. (12) Several studies have correlated elevated intraoperative LAP with increased risk of recurrent AF after ablation. (7,13-17) Elevated LAP also correlates with symptomatic HF but few studies have examined the relationship between early incident HF and LAP during AF ablation. (18) The aim of our study is to examine how LAP before and after ablation affects postoperative HF. 


\section{METHODS}

\section{Study Design}

To better assess the relationship between AF ablation and postoperative HF, we first conducted a retrospective study of all patients undergoing AF ablation at St Vincent Hospital (Indianapolis, IN, USA) from March 1, 2017 to September 30, 2018. Medical records were examined to ascertain HF incidence in the 30day postoperative period. International Classification of Disease, version 10 codes for clinical heart failure were used to ascertain the primary endpoint, which was gleaned from telephone encounters, office visits, emergency room visits, or hospitalizations (see supplement table 1).

After analysis of the retrospective cohort, we then designed a prospective, single center, cohort study examining 100 consecutive patients undergoing AF ablation at our institution from February 2019 to June 2019. Excluded patients were unable or unwilling to consent, could not have LAP successfully measured, or did not complete follow-up. The study protocol was approved by the Institutional Review Board and received proper ethical oversight. Informed consent was obtained from every subject because of the prospective nature of the study. No interventions were mandated by the study protocol based on the information obtained. Baseline characteristics and procedural data were collected at the time of the procedure via the electronic medical record and procedural reports. Patients were followed for a 30-day period. At the end of follow-up, each patient received a prespecified phone survey to assess for any clinical outcomes (see supplemental figure 1).

\section{Catheter Ablation}

Catheter Ablation (CA) was performed by 5 experienced electrophysiologists. Some patients underwent preprocedural imaging with cardiac computed tomographic imaging or cardiac magnetic resonance imaging while others did not. Each patient was anticoagulated without interruption or had preprocedural transesophageal echocardiogram or intracardiac echocardiogram to exclude cardiac thrombus prior to CA. All CA were assisted by 3-dimensional electroanatomic mapping with either CARTO 3v6 (Biosense Webster, Irvine, CA) or Ensite Precision (Abbott, Abbott Park, IL). Patients were placed under general anesthesia and either single or double transseptal puncture was performed with the aid of intracardiac ultrasound. Procedures utilizing CARTO mapping used the Pentaray (Biosense, Irvine, CA) mapping catheter with the Thermocool Smarttouch SF ablation catheter (Biosense, Irvine, CA). Procedures utilizing Ensite Precision used either the Reflexion Spiral or Advisor HD Grid for mapping and Tacticath ablation catheter (Abbott, Abbott Park, IL). Pulmonary vein isolation was performed with radiofrequency ablation at 30-50 W and confirmed by achieving entrance and exit block. Additional targeted ablations (caval tricuspid isthmus, posterior box, roof line, anterior mitral line and isolation of the left atrial appendage) were performed at the discretion of the performing electrophysiologist based on each subject's unique clinical history. Focal impulse and rotor modulation mapping and ablation with a 64 pole basket catheter (Topera, Abbott, Palo Alto, CA) was also performed when deemed appropriate. Right and left atrial pressures were documented pre and post ablation. Pre ablation pressure was measured in their presenting rhythm while the post ablation pressure as measured in normal sinus rhythm. Anticoagulation was resumed post procedure for a minimum of 2 months. Antiarrhythmic drugs were stopped 5 half-lives prior to ablation in all cases except with amiodarone, which was stopped 14 days prior to ablation. Diuretics were held on the day of the procedure. Post ablation antiarrhythmics were discontinued in most cases. Accurate intake and output during the procedure and during the index hospitalization were documented. The use of bladder catheters was limited based on data describing an increased incidence of urinary complications. (20) Routine post procedure care was employed without mandating any clinical changes to patient's care based on data obtained from the research study. Patients were followed for 30 days.

\section{Collection of procedural variables}

Transseptal access was achieved in the standard fashion using a BRK needle (Abbott, Abbott Park, IL) and a long sheath (typically a medium curl Agilis or SL1 [both Abbott, Abbott Park, IL]). Baseline variables were collected before and after initial transseptal access ("pre" ablation values). Pressure readings were made after zeroing the pressure line with the transducer at the level of the inferior vena cava. Ablation 
was performed as described above, and those in persistent $\mathrm{AF}$ were cardioverted after ablation. After the waiting period just prior to sheath removal, measurements were collected again ("post" ablation values), including both left atrial and right atrial pressures. Mean values over a 10 second window were used without regard to the respiratory cycle. All patients received general anesthesia with standard ventilation parameters. Cardiac output was calculated using Fick's equation and a right atrial sampling for the mixed venous oxygen saturation.

\section{Outcomes}

The primary outcome was acute HF event rate within 30 days. HF was defined as any clinical encounter that invoked a HF exacerbation, including any telephone encounter, office visit, emergency room visit, hospitalization, or positive response to the 30-day follow-up survey (see supplemental figure 1). For the purposes of this study, diuretic medication adjustment was required to meet the primary endpoint. The secondary outcome was rate of AF-free HF events within 30 days.

\section{Statistical Analysis}

Statistical analysis was performed using SPSS v 25 (IBM corporation, Armonk, NY). All missing data was excluded and outliers were included. Categorical variables were compared using Chi-squared test or Fisher exact test while continuous variables were compared using student's t-test or Mann-Whitney test for normally or non-normally distributions respectively. Univariate regression was used to compare the primary endpoint with baseline variables. Multivariate analysis was performed using multi nominal regression analysis, with step-wise inclusion into the model. Two tailed $\mathrm{P}$ values $<0.05$ were considered statistically significant. An exploratory analysis of the best discriminators of AF-free HF events was performed using a receiver-operator curve.

To determine sample size for the prospective cohort, we assumed a doubling of the primary endpoint compared to the retrospective cohort given the prospective ascertainment. Additionally, based on previous data that acute hemodynamic changes affect clinical $\mathrm{HF}$, we assumed a left atrial pressure difference of $5 \mathrm{mmHg}$ in those with or without early incident $\mathrm{HF}(14+/-6 \mathrm{mmHg}$. $9 \mathrm{mmHg})$, and with alpha $=0.05$ and beta $=$ 0.80, a total of 104 patients was recommended (https://clincalc.com/stats/samplesize.aspx). (21) Because of the greater than expected rate of HF, the study was stopped at 100 subjects.

\section{RESULTS}

For the retrospective analysis, there were 391 patients who were included, with a primary endpoint reached in 13 patients (3.3\%). When the group of early incident HF patients was compared to the control group, univariate correlates to the primary endpoint included longer procedure time (5:47 v. $4: 41 \mathrm{hr}, \mathrm{p}=0.002)$, greater fluid administration (2406 $\mathrm{v} 1887 \mathrm{~mL}, \mathrm{p}=0.015)$, and preexisting diagnosis of $\mathrm{HF}(69 \% \mathrm{v} 39 \%, \mathrm{p}=$ 0.03). Persistent AF, redo procedure, gender, age, body mass index, chronic kidney disease, serum creatinine, diabetes mellitus, and coronary disease were not different in the two groups. Significantly associated variables were included in a multivariable model, but no factors remained independently associated with the primary outcome.

\section{Prospective Study}

For the prospective study, we enrolled 100 consecutive patients undergoing AF ablation. No patient was excluded or lost to follow-up. A HF episode occurred in $20 \%$ of the patients within 30 days. Fourteen patients out of the $20(70 \%)$ had a HF episode captured by the prespecified phone survey administered at 30 days. Three patients (15\%) had a HF episode captured via electronic medical record review and 3 patients (15\%) had a HF episode captured by both methods (Figure 1).

Baseline demographics and procedural details are displayed in Table 1 . The average age was $64.5 \pm 10$ and $63 \%$ of the subjects were males. The average ejection fraction was $50 \pm 10 \%$, with HF prevalence of $17 \%$, a group whose mean $\mathrm{EF}$ was $46 \pm 11 \%$. Baseline comorbidities and procedural characteristics were similar between those with or without early incident HF with few exceptions. Patients that had a HF episode had 
a higher incidence of moderate to severe mitral valve disease (20\% vs $4 \% ; \mathrm{p}=0.01)$. They were also more likely to have persistent $\mathrm{AF}(70 \% \mathrm{v} 41 \% ; \mathrm{p}=0.02)$, prescribed a loop diuretic $(40 \% \mathrm{v} 17.5 \%$; $=0.03)$, have a higher LAP post-ablation ( $12.6 \mathrm{v} 10.2 \mathrm{mmHg} ; \mathrm{p}=0.04)$, and recurrence of AF within 30 days $(55 \% \mathrm{v} 14 \%$; $\mathrm{p}=0.01$ ). Patients that did not have a HF episode were more likely to be taking class III antiarrhythmic medications preoperatively ( $44 \% \mathrm{v} 15 \% ; \mathrm{p}=0.018)$. Additionally, those who were taking antiarrhythmics after ablation had a lower incidence of HF $(22.5 \% \mathrm{v} 0 \% ; \mathrm{p}=0.019)$. There was no difference in use of beta blocker, calcium channel blockers or class I antiarrhythmic medication between the two groups. Most patients had class I and class III antiarrhythmics held after ablation (95\% and $72 \%$ respectively) though class II and IV antiarrhythmics remained at pre procedure doses.

\section{Multivariate Analysis}

A multivariate analysis was performed (Table 2 ) using moderate to severe mitral valve disease, use of class III antiarrhythmics, use of loop diuretics, persistent AF, LAP, and 30-day AF recurrence. Patients with moderate to severe mitral valve disease (odds ratio (OR) 8.7 [95\% confidence interval $3.3-22.6]$; p $=0.03)$ and taking a loop diuretic (OR $4.8[2.6-9.1] ; \mathrm{p}=0.01)$ were more likely to have a HF event. Those taking class III antiarrhythmics were less likely to have a HF event (OR $0.24[0.1-0.5] ; \mathrm{p}=0.04)$ as seen in theFigure 2. A second multivariate analysis incorporating variable associated with HF events in the retrospective study (procedure time, fluid administration and preexisting HF diagnosis) revealed no persistent relationship with HF events. Finally, a third multivariate analysis was performed that included demographic information putatively associated with HF events. Of these variables (Table 3 ), only the presence of mitral valve disease was significant (OR $6.6[1.3-34] ; \mathrm{p}=0.03$ ).

\section{AF-free $H F$}

Of the 20 patients that had a HF event, 9 did not have a recurrence of AF. All baseline demographics and procedural details were compared between those that had an AF-free HF event and those that did not. A univariate analysis revealed patients with chronic kidney disease (CKD), higher LAP, and higher RAP post-procedure were more likely to have an AF-free HF event (Table 4 ). Here, because of the small sample size, values were not normally distributed. Median (interquartile range) LAP and RAP for those without a HF event compared to those with a HF event were $9.5(7,13)$ v $14(9,15.5)$ and $5(4,7.8)$ v $9(5.5,11)$ $\mathrm{mmHg}$, respectively. Unlike patients with a HF event and recurrent AF, the presence of mitral valve disease, use of a loop diuretic, persistent AF and lack of class III antiarrhythmic did not correlate to the endpoint of AF-free HF. A receiver-operatory curve was performed and identified the sum of the LAP and RAP as a discriminator of AF-free HF events (Figure 3 ). Specifically, those patients with a LAP and RAP sum greater than or equal to 20 were more likely to have an $\mathrm{AF}$-free $\mathrm{HF}$ event.

\section{DISCUSSION}

Ablation has proven to be a safe and effective treatment option for patients with AF. Over the past several years, studies have revealed that the incidence of HF post-ablation is much higher than initially described. Our retrospective study only found a $3.3 \%$ incidence of early postop HF. Those most susceptible were, unsurprisingly, those with a known diagnosis of HF, and those who received more intraprocedural fluid and had longer cases. These variables were codependent, as the multivariable model showed no independent associations with HF. However, in our prospective, single center study with perfect 30 day follow up, we confirmed other recent data by reporting a $20 \%$ incidence of HF post-ablation. Incident HF may be related to recurrent AF, baseline loop diuretic use and elevated atrial pressures, especially in those with moderatesevere mitral valve disease and a history of persistent AF. The rate difference compared to the retrospective cohort confirms a large underascertainment from retrospective studies, and the importance of prospective data collection in this population.

\section{Multivariate Analysis}

Our multivariate analysis suggests AF recurrence is a risk factor for HF events post ablation. In fact, the use of a class III antiarrhythmic to maintain sinus rhythm post-ablation may even be protective against 
HF. These patients also had an increased LAP post-ablation but this was not associated with increased HF events in a multivariate analysis. Kishima et al previously described elevated LAP is a risk factor for developing AF post-ablation. (14) We expand upon this and propose that a recurrence of AF produces lack of atrial contribution to cardiac output, decreases ventricular filling with tachycardia and disrupts intracellular calcium handling precipitating HF events. Though cardiac output, heart rate, and systemic blood pressure measured acutely during the procedure did not correlate to incident HF, post ablation LAP may uniquely reflect the ability of the atrium to handle volume in the setting of inflammatory changes from ablation. Taking steps to prevent AF recurrence post-ablation may mitigate the adverse pathophysiologic effects of $\mathrm{AF}$ and reduce HF events. Furthermore, the use of a loop diuretic pre-procedurally may be an indicator of susceptibility to hypervolemic states such as HF.

\section{AF-free HF Events}

Recurrent AF post-ablation may be a risk factor for developing HF but this only accounts for about half of the HF events in our study population. In the remaining patients, we found the presence of CKD and elevated RAP and LAP post-ablation correlated with AF-free HF events. Elevated atrial pressures, if not appropriately reduced, likely results in pulmonary vascular congestion and HF symptoms. This may provide a unique marker for patients at risk of developing a subsequent HF episode and provide an opportunity for intervention. A reasonable trigger for intervention may be the sum of the LAP and RAP greater than or equal to $20 \mathrm{mmHg}$ based on this data (encompassing $77.8 \%$ of those with incident HF without a recurrence of $\mathrm{AF}$ ), but further study is warranted for validation, as this would apply to $33 \%$ of the entire population.

\section{Ascertainment Bias}

The range of incident HF could be from $6 \%$ (chart review only) to $20 \%$ (chart review plus telephone survey). Similar to Huang et al, we performed a prospective study that included a prespecified telephone survey at the end of follow-up. (7) One limitation of previous, retrospective studies is underascertainment of the primary endpoint.

\section{LIMITATIONS}

Given this was a single-center prospective study, the results may lack generalizability. The study protocol did not require standardization of ablation strategies. Though procedure time and fluid administration may differ based on operator and ablation strategy, these parameters were not significantly associated with the primary outcome. Some echocardiographic parameters, such as diastolic dysfunction, were not available prior to the procedure. However, diastolic dysfunction aims to estimate left atrial filling pressures, which was measured directly in our study. Finally, the telephone survey ascertained over half of HF episodes, whose clinical relevance is unclear, though in all positive cases diuretic adjustment had been made.

\section{CONCLUSION}

The current study reports early incident HF may complicate up to $20 \%$ of AF ablation procedures. The majority of adverse events was associated with recurrent AF post-ablation. However, in those without a recurrence of AF post-ablation, elevated RAP and LAP post-ablation was associated incident HF events. Future, prospective randomized trials are needed to elucidate if reducing elevated atrial pressure post-ablation can reduce HF events.

\begin{tabular}{lllll}
\hline & All Patients $(\mathrm{n}=100)$ & No HF $(\mathrm{n}=80)$ & HF $(\mathrm{n}=20)$ & P-value \\
\hline Age & $64.5 \pm 10.7$ & $64.3 \pm 10.7$ & $65.3 \pm 10.7$ & 0.706 \\
Male & $63(63 \%)$ & $52(65 \%)$ & $11(55 \%)$ & 0.413 \\
Weight (kg) & $94.3 \pm 19.2$ & $94.7 \pm 19.5$ & $92.6 \pm 13.5$ & 0.669 \\
Body Mass Index & $30.5 \pm 5.3$ & $30.3 \pm 5.4$ & $31.1 \pm 3.9$ & 0.546 \\
Congestive Heart Failure & $17(17 \%)$ & $13(16 \%)$ & $4(20 \%)$ & 0.693 \\
Ejection Fraction (Mean \%) & $52 \% \pm 10.4 \%$ & $51.5 \% \pm 11 \%$ & $53.6 \% \pm 8.3 \%$ & 0.488 \\
Diabetes Mellitus & $17(17 \%)$ & $12(15 \%)$ & $5(25 \%)$ & 0.292
\end{tabular}




\begin{tabular}{lllll}
\hline & All Patients $(\mathrm{n}=100)$ & No HF $(\mathrm{n}=80)$ & HF $(\mathrm{n}=20)$ & P-value \\
\hline Hypertension & $61(61 \%)$ & $47(59 \%)$ & $14(70 \%)$ & 0.361 \\
Coronary Artery Disease & $24(24 \%)$ & $17(21 \%)$ & $7(35 \%)$ & 0.202 \\
Chronic Kidney Disease & $6(6 \%)$ & $4(5 \%)$ & $2(10 \%)$ & 0.405 \\
Moderate to Severe Mitral Disease & $7(7 \%)$ & $3(4 \%)$ & $4(20 \%)$ & 0.01 \\
Beta Blocker & $60(60 \%)$ & $45(56 \%)$ & $15(75 \%)$ & 0.128 \\
Calcium Channel Blocker & $30(30 \%)$ & $25(31 \%)$ & $5(25 \%)$ & 0.590 \\
Class I Antiarrhythmic Pre-Ablation & $6(6 \%)$ & $4(5 \%)$ & $2(10 \%)$ & 0.405 \\
Class I Antiarrhythmic Post-Ablation & $5(5 \%)$ & $4(5 \%)$ & $1(5 \%)$ & 1 \\
Class III Antiarrhythmic Pre-Ablation & $42(42 \%)$ & $38(47.5 \%)$ & $4(20 \%)$ & 0.026 \\
Class III Antiarrhythmic Post-Ablation & $18(18 \%)$ & $18(22.5 \%)$ & $0(0 \%)$ & 0.019 \\
Loop Diuretic Pre-Ablation & $22(22 \%)$ & $14(17.5 \%)$ & $8(40 \%)$ & 0.03 \\
Loop Diuretic Post-Ablation & $19(19 \%)$ & $13(16.3 \%)$ & $6(30 \%)$ & 0.161 \\
Intake During Procedure (mL) & $1469.4 \pm 808$ & $1439 \pm 806.5$ & $1589.4 \pm 889.1$ & 0.460 \\
Output During Procedure (mL) & $66.8 \pm 239.3$ & $73.4 \pm 246.5$ & $41.3 \pm 0$ & 0.595 \\
Intake During Hospital Stay (mL) & $265.8 \pm 386.4$ & $280.3 \pm 397$ & $208.7 \pm 160.2$ & 0.462 \\
Output During Hospital Stay (mL) & $152.4 \pm 594.1$ & $119.6 \pm 611.9$ & $283.8 \pm 81.6$ & 0.271 \\
Hemoglobin (mg/dL) & $14.2 \pm 2$ & $14.2 \pm 2$ & $14.3 \pm 1.1$ & 0.889 \\
Systolic Blood Pressure Post-Ablation & $104.6 \pm 15.7$ & $104.4 \pm 16.4$ & $105.6 \pm 13.1$ & 0.779 \\
Diastolic Blood Pressure Post-Ablation & $59.7 \pm 11.1$ & $59.8 \pm 11.5$ & $59.4 \pm 9.7$ & 0.870 \\
Heart Rate Post-Ablation & $69.4 \pm 17.4$ & $70.6 \pm 16.8$ & $64.7 \pm 19$ & 0.191 \\
Cardiac Output & $8.8 \pm 9.3$ & $8.9 \pm 10.1$ & $8.3 \pm 4.4$ & 0.142 \\
Left Atrial Pressure Pre-Ablation (mmHg) & $11.4 \pm 5.2$ & $11.0 \pm 4.7$ & $12.7 \pm 10.4$ & 0.20 \\
Right Atrial Pressure Pre-Ablation (mmHg) & $7 \pm 4$ & $6.9 \pm 3.8$ & $7.6 \pm 6.1$ & 0.171 \\
Left Atrial Pressure Post-Ablation (mmHg) & $10.6 \pm 4.4$ & $10.2 \pm 4.3$ & $12.6 \pm 4.9$ & 0.04 \\
Right Atrial Pressure Post-Ablation (mmHg) & $6.1 \pm 3.2$ & $5.7 \pm 3.1$ & $7.6 \pm 4.1$ & 0.06 \\
Persistent Atrial Fibrillation & $46(46 \%)$ & $32(41 \%)$ & $14(70 \%)$ & 0.02 \\
30-Day Atrial Fibrillation Recurrence & $22(22 \%)$ & $11(14 \%)$ & $11(55 \%)$ & 0.01 \\
\hline
\end{tabular}

Table 1: Baseline demographics and univariate analysis of variables associated with HF events. Values are presented as median \pm standard deviation or number $(\%)$.

\begin{tabular}{llll}
\hline & Univariate Analysis & Multivariate Analysis & Multivariate Analysis \\
\hline & P Value & P Value & Odds Ratio (95\% CI) \\
Left Atrial Pressure Post-Ablation (mmHg) & 0.04 & 0.21 & $1.1(1-1.2)$ \\
Moderate to Severe Mitral Valve Disease & 0.01 & 0.03 & $8.7(3.3-22.6)$ \\
Class III Antiarrhythmic Pre-Ablation & 0.03 & 0.04 & $0.2(0.1-0.5)$ \\
Loop Diuretic Pre-Ablation & 0.03 & 0.01 & $4.8(2.6-9.1)$ \\
Persistent Atrial Fibrillation & 0.02 & 0.43 & $0.6(0.2-2.1)$ \\
\hline
\end{tabular}

Table 2: Multivariate analysis of significant variables based on univariate analysis. All variables were significant based on univariate analysis. However, on multivariate analysis only moderate to severe mitral valve disease, class III antiarrhythmic use pre-ablation and loop diuretic pre-ablation remained significant.

\begin{tabular}{lll}
\hline & Multivariate Analysis & Multivariate Analysis \\
\hline & P Value & Odds Ratio $(95 \% \mathrm{CI})$ \\
Total Procedural Fluid & 0.35 & $1(1-1)$ \\
Congestive Heart Failure & 0.66 & $0.72(0.2-3.1)$
\end{tabular}




\begin{tabular}{lll}
\hline & Multivariate Analysis & Multivariate Analysis \\
\hline Chronic Kidney Disease & 0.55 & $0.54(0.1-4.1)$ \\
Mitral Valve Disease & 0.03 & $6.6(1.3-34)$ \\
Any Antiarrhythmic Pre & 0.08 & $2.8(0.9-8.8)$ \\
\hline
\end{tabular}

Table 3. Multivariate analysis of putative demographics associated with HF events. Mitral valve disease was the only variable that remained significant with a multivariate analysis.

\begin{tabular}{llll}
\hline & No AF-Free HF $(\mathrm{n}=91)$ & AF-Free HF $(\mathrm{n}=9)$ & P Value \\
\hline Left Atrial Pressure Post-Ablation $(\mathrm{mmHg})$ & $9.5(7,13)$ & $14(9,15.5)$ & 0.049 \\
Right Atrial Pressure Post-Ablation $(\mathrm{mmHg})$ & $5(4,7.8)$ & $9(5.5,11)$ & 0.016 \\
Chronic Kidney Disease & $4(4 \%)$ & $2(22 \%)$ & 0.032 \\
\hline
\end{tabular}

Table 4: Univariate analysis of variables associated with AF-Free HF events. Variable that were significantly different between the two groups on univariate analysis. No variable remained significant after multivariate analysis.

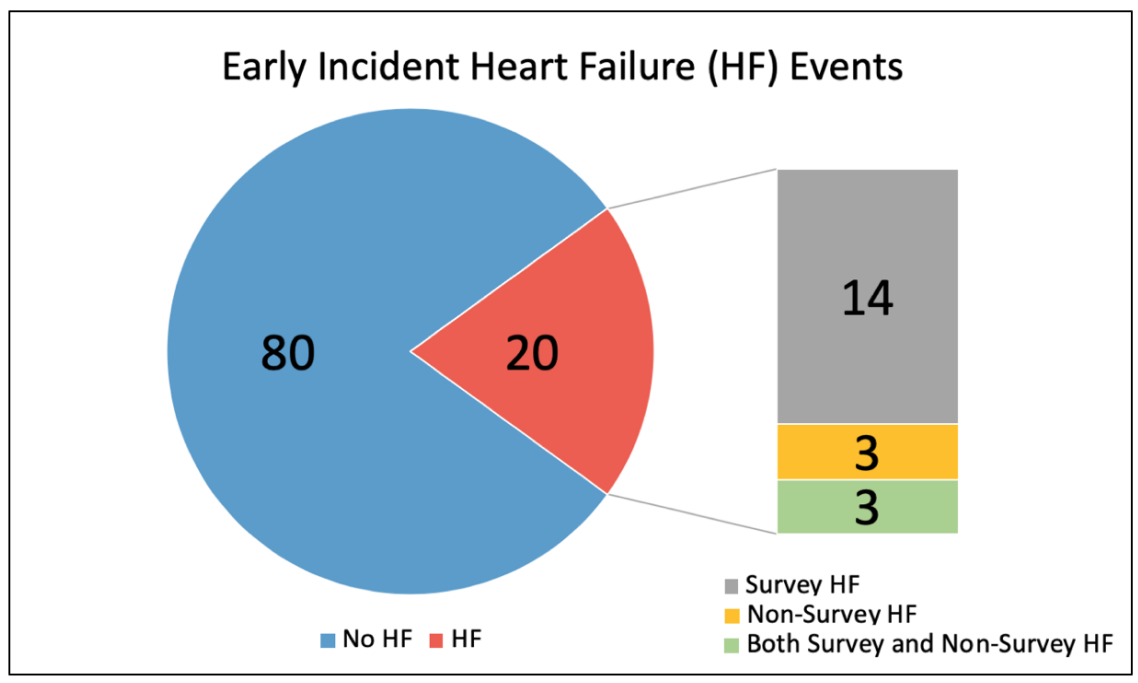

Figure 1: Early incident heart failure (HF) events. Out of the 100 patients, 20 had a HF event during follow-up. Fourteen patients had their HF event identified from the pre-specified survey. Three patients had a HF event identified without a survey and 3 patients were identified by both means. 


\section{Odds Ratio For Incident HF Event}

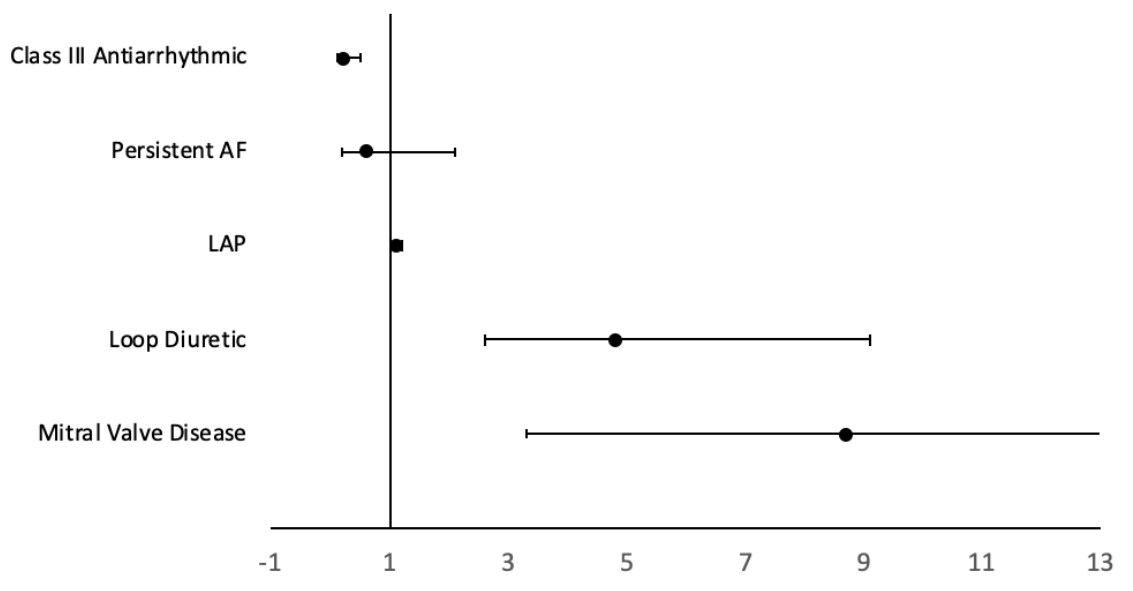

Figure 2: Odds ratio for incident HF event. Moderate to severe mitral valve disease and chronic loop diuretic are associated with increased risk of heart failure (HF) events with an odds ratio (OR) (95\% confidence interval) of $8.7(3.3-22.6)$ and $4.8(2.6-9.1)$ respectively. Class III antiarrhythmic use was associated with a reduced risk of $\mathrm{HF}$ events with an OR of $0.2(0.1-0.5)$. Left atrial pressure (LAP) post ablation and persistent atrial fibrillation (AF) were not associated with HF events with an OR of $1.1(1-1.2)$ and 0.6 $(0.2-2.1)$ respectively. 


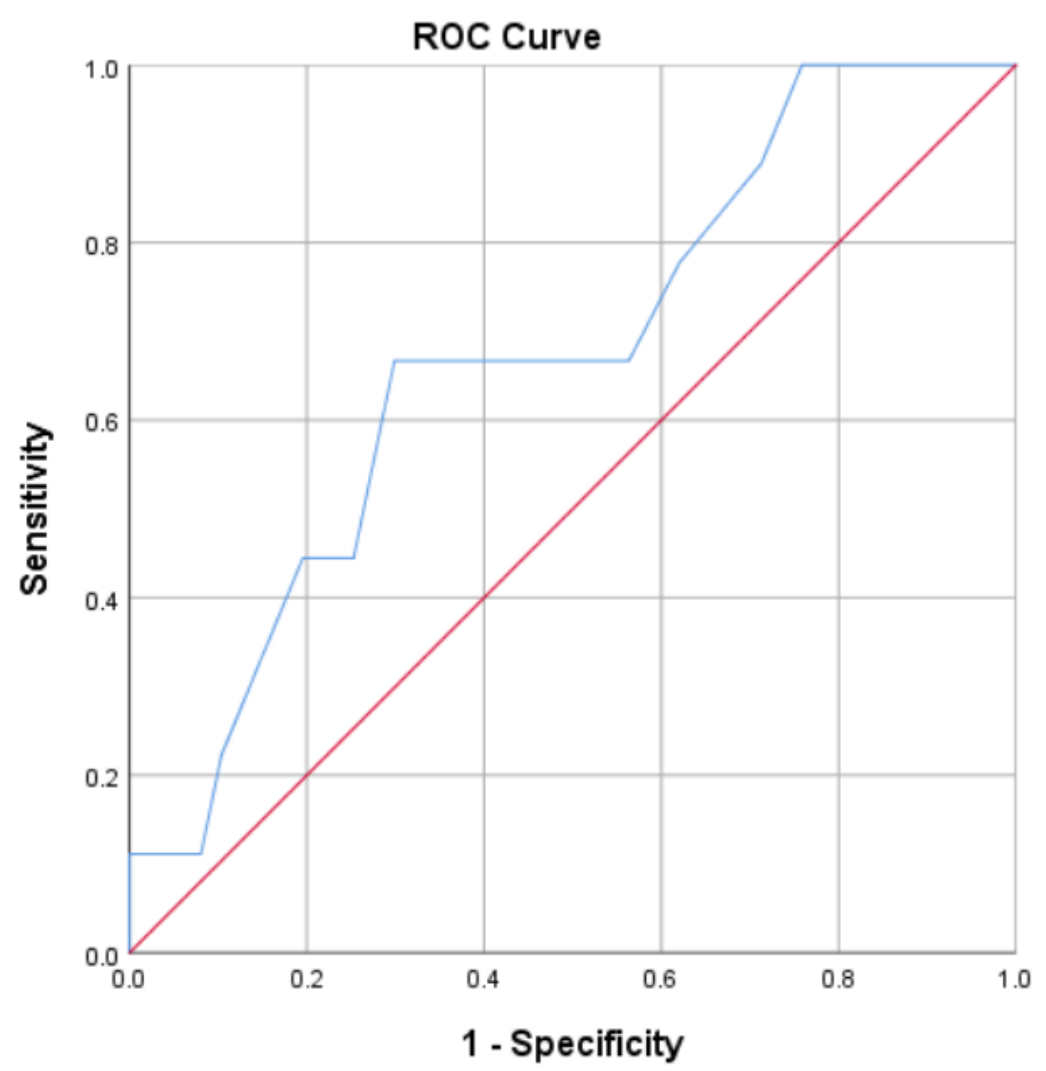

Figure 3: Receiver-operator curve for the sum of the atrial pressures and occurrence of atrial fibrillation-free heart failure events.

\section{REFERENCES}

1. Benjamin EJ., et al., Impact of atrial fibrillation on the risk of death: the Framingham Heart Study. Circulation 1998; 98:946 - 52.

2. Ball J, Carrington MJ, McMurray JJ, Steart S. Atrial fibrillation: profile and burden of an evolving epidemic in the 21st century. Int J Cardiol 2013;167:1807 - 24.

3. Wazni, O.M., et al., Radiofrequency ablation vs antiarrhythmic drugs as first-line treatment of symptomatic atrial fibrillation: a randomized trial. JAMA, 2005. 293 (21): p. 2634-40.

4. Wilber, D.J., et al., Comparison of antiarrhythmic drug therapy and radiofrequency catheter ablation in patients with paroxysmal atrial fibrillation: a randomized controlled trial. JAMA, 2010.303 (4): p. $333-40$

5. Arora, S., et al., Causes and Predictors of Readmission in Patients With Atrial Fibrillation Undergoing Catheter Ablation: A National Population-Based Cohort Study. J Am Heart Assoc, 2018.7 (12).

6. Garg, J., et al., Predictors of 30-day readmissions after catheter ablation for atrial fibrillation in the USA. J Interv Card Electrophysiol, 2019.

7. Huang, H.D., et al., Incidence and risk factors for symptomatic heart failure after catheter ablation of atrial fibrillation and atrial flutter. Europace, 2016. 18 (4): p. 521-30.

8. Ikenaga, H., et al., Radiofrequency catheter ablation is effective for atrial fibrillation patients with hypertrophic cardiomyopathy by decreasing left atrial pressure. J Arrhythm, 2017.33 (4): p. 256-261.

9. Joy, P.S., R. Gopinathannair, and B. Olshansky, Effect of Ablation for Atrial Fibrillation on Heart Failure Readmission Rates.Am J Cardiol, 2017. 120 (9): p. 1572-1577. 
10. Srivatsa, U.N., et al., California Study of Ablation for Atrial Fibrillation:Re-hospitalization for Cardiac Events (CAABL-CE). J Atr Fibrillation, 2018. 11 (1): p. 2036.

11. Maisel WH, Stevenson LW. Atrial fibrillation in heart failure: epidemiology, pathophysiology, and rationale for therapy. Am J Cardiol . 2003; 91:2D-8D.

12. Anter, E., et al., Atrial Fibrillation and Heart Failure Treatment Considerations for a Dual Epidemic. Circ 2009. 119: p. 2516-25.

13. Evranos, B., et al., Increased left atrial pressure predicts recurrence following successful cryoablation for atrial fibrillation with second-generation cryoballoon. J Interv Card Electrophysiol, 2016. 46 (2): p. $145-51$.

14. Kishima, H., et al., The Impact of Elevated Left Atrial Pressure in Sinus Rhythm After Cardioversion on Outcomes After Catheter Ablation for Atrial Fibrillation. J Cardiovasc Electrophysiol, 2016.27 (7): p. 813-9.

15. Kishima, H., et al., Left atrial pressure pattern without a-wave in sinus rhythm after cardioversion affects the outcomes after catheter ablation for atrial fibrillation. Heart Vessels, 2018.33 (11): p. 1365-1372.

16. Park, J., et al., Low Left Atrial Compliance Contributes to the Clinical Recurrence of Atrial Fibrillation after Catheter Ablation in Patients with Structurally and Functionally Normal Heart. PLoS One, 2015. 10 (12): p. e0143853.

17. Sramko, M., et al., Resting and Exercise-Induced Left Atrial Hypertension in Patients With Atrial Fibrillation: The Causes and Implications for Catheter Ablation. JACC Clin Electrophysiol, 2017.3 (5): p. 461-469.

18. Poole-Wilson PA, Buller NP. Causes of symptoms in chronic congestive heart failure and implications for treatment. American Journal of Cardiology 1988. 62 31A-34A.

19. Lehman, A., et al., Avoiding Urinary Catheterization in Patients Undergoing Atrial Fibrillation Catheter Ablation. J Afib, 2019-2020.12 (4): p. 12-15.

20. Ahmed, A., et al., Avoiding Bladder Catheterization During Atrial Fibrillation Ablations. J Am Coll Cardiol EP, 2020.6 (2): p. 185-190.

21. Lal, s., et al,. Acute Haemodynamic Effects of Frusemide in Patients with Normal and Raised Left Atrial Pressure. Brit Heart J 196931 : p. 711-717. 